\title{
Principios de la Formación Especializada del Director Escolar. Una Propuesta desde la Sistematización
}

\section{Principles of the Specialized Formation of the School Principal. A Proposal from the Systematization}

\author{
Pedro Valiente Sandó * \\ José Javier Del Toro Prada \\ Jorge González Ramírez \\ Universidad de Holguín
}

\begin{abstract}
La formulación de un sistema de principios de la formación especializada del director escolar, para explicar teóricamente dicho proceso y ofrecer orientación metodológica que viabilice su realización práctica, constituyó el objetivo de una investigación cuya fundamentación teórica, concepción metodológica y resultados se exponen en este artículo. La estrategia metodológica para alcanzar dicho objetivo fue la sistematización de la experiencia teórica precedente relativa a la formación de los directores escolares, otros profesionales de la educación, directivos empresariales y otros grupos de recursos laborales, mediante el examen exhaustivo de una vasta literatura. Los principios establecidos fueron: a) el principio del vínculo entre la formación inicial del educador y la formación especializada (inicial y permanente) para la dirección escolar; b) el principio de la condicionalidad entre los referentes orientadores y la pertinencia social de la formación; c) el principio del enfoque sistémico, progresivo y planeado de la formación; d) el principio de la relación entre el contenido contextualizado de la formación, el trabajo cooperado en grupo y la atención diferenciada en el proceso formativo, y; e) el principio de la relación entre la sistematicidad de la evaluación y el mejoramiento cualitativo del proceso y los resultados de la formación.
\end{abstract}

Descriptores: Principios, Formación especializada, Dirección escolar, Sistematización.

The formulation of a system of principles for the specialized formation of the school principal, in order to explain this process theoretically and to offer methodological orientations that help putting this into practice, was the main concern of a research task; its theoretical framework, the methodological conception, and results are included in this article. The methodological strategy followed to reach such objective was the systematization of the preceding theoretical experience related to the formation of school principals, other professionals of education, enterprise executives, and other working staff. The systematization process involved a detailed revision of a wide range of bibliographical sources. The established principles are the following: a) the link between the initial formation of the educator and the specialized formation (initial and permanent) for school leadership, b) the conditioned character between the orientation referents and the social pertinence of the formation, c) the systemic, progressive, and planned approach of the formation, d) the relation between the contextualized content of the formation, the cooperative group work, and the attention to individual differences in the formative process, and e) the relation between the systematic character of evaluation and the qualitative improvement of the process and the results of the formation.

Keywords: Principles, Specialized formation, School leadership, Systematization.

\begin{tabular}{|c|c|c|}
\hline *Contacto: pvaliente@facii.uho.edu.cu & Recibido: & 7 de enero 2015 \\
\hline 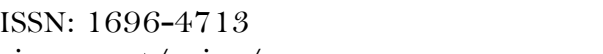 & $1^{a}$ Evaluación: & 10 de marzo 2015 \\
\hline e.net/reice/ & $2^{\text {a }}$ Evaluación: & 2 de mayo 2015 \\
\hline stas.uam.es/reice & Aceptado: & 3 de junio 2015 \\
\hline
\end{tabular}




\section{Introducción}

En las últimas décadas ha crecido el consenso sobre la necesidad de la formación especializada del director escolar (FEDE), en asociación con el reconocimiento del carácter profesional especializado de su actividad y de la consiguiente necesidad de su profesionalización y especialización, devenida del impacto que han tenido las reformas educativas, que han planteado nuevas exigencias a la actividad de este directivo educacional; así como de la especificidad de dicha actividad que, en las nuevas circunstancias, aumenta su complejidad.

La FEDE, a la que corresponde un papel decisivo en su profesionalización y especialización, puede entenderse como un proceso formal, intencional, consciente y dirigido, como un sistema de interinfluencias, y como el resultado del efecto sistemático y coherente de un conjunto de actividades organizadas, encaminadas a la adquisición y desarrollo de los conocimientos, habilidades y valores que posibilitan la conformación y consolidación de las competencias directivas que lo hacen apto para el desempeño adecuado de su actividad profesional de dirección (APD).

En Cuba, la década de los noventa del siglo veinte fue el punto de despegue para las investigaciones sobre este objeto y el desarrollo de acciones prácticas de formación que, basadas en la experiencia precedente, comenzaron a aprovechar los valiosos resultados teóricos y prácticos que se han ido obteniendo.

Ante la necesidad de comenzar a sistematizar los resultados investigativos alcanzados y estudiar experiencias investigativas desarrolladas en otros países, especialmente de Iberoamérica, desde el año 2012 se desarrolla el Proyecto de Investigación "Formación del Director Escolar", auspiciado por la Universidad de Holguín, del que participan los autores de este artículo. El proyecto está dirigido a la sistematización de una concepción teórico-metodológica de la FEDE, que comprende fundamentos conceptuales generales, principios y referentes orientadores de dicha formación; así como una caracterización de los procesos pedagógico y directivo en que ella se concreta.

Los principios que se exponen en este artículo ocupan una posición especial de orientación teórico-metodológica dentro de la citada concepción. Coadyuvan a su sustentación en el orden teórico y constituyen una guía orientadora para la realización práctica de la formación como proceso que tiene naturaleza pedagógica y directiva.

\section{Fundamentación Teórica}

En la ciencia, la formulación y fundamentación de principios revela el alcance de mayores niveles de esencialidad en el conocimiento teórico de los objetos y fenómenos. Los principios son:

... ideas centrales, conceptos generales, punto de partida en la estructuración y exposición de la teoría científica, los que se convierten en la primera determinación de las ideas de una teoría y cumplen una función metodológica importante a la hora de explicar un nuevo conocimiento. (Kopnin, 1983, p. 383)

Los principios pueden cumplir dicha función metodológica, al ser contentivos de reglas y/o precisar ciertas implicaciones que deben tenerse en cuenta al momento de su aplicación. Tienen, por consiguiente, una relevante importancia práctica al posibilitar 
que el conocimiento teórico sistematizado pueda ser utilizado en la optimización de los procesos que explican.

Por ser la FEDE un objeto de estudio joven son muy escasas y limitadas las propuestas específicas de principios, u otros postulados teóricos de carácter general, que puedan servir para explicar dicho objeto en su esencialidad y ofrecer pautas para su ejecución como proceso práctico.

En estas circunstancias, la formulación de una propuesta al respecto debe considerar entre sus antecedentes de mayor relevancia los estudios relacionados con la formación y desarrollo de los directores escolares, otros profesionales de la educación, directivos empresariales y otros grupos de recursos laborales, realizados en Cuba y otros países, algunos de los cuales incluyen el planteamiento de principios, u otros postulados teóricos de carácter general, que resultan valiosos para develar, desde la perspectiva teórica, relaciones esenciales presentes en el proceso de formación de los directores escolares.

En la literatura sobre Administración que se consultó son escasos los aportes que, en calidad de generalizaciones, expresen relaciones esenciales acerca de la formación y desarrollo de los recursos humanos, como objeto de estudio, y orienten teórica y metodológicamente su realización como proceso. No obstante, los autores precisan un conjunto de ideas esenciales de gran utilidad para formular una propuesta de principios de la FEDE.

Koontz y Weihrich (2008) identifican tres principios que atañen específicamente a la formación y desarrollo de los directivos:

- Principio de la capacitación y el desarrollo de los administradores, que postula: "Cuanto más se integren la capacitación y el desarrollo de los administradores con el proceso administrativo y los objetivos de la empresa, más eficaces serán los programas y actividades de desarrollo" (p. 758).

- Principio de los objetivos de capacitación, que establece: "A mayor precisión en la formulación de los objetivos de capacitación, más probabilidades habrá de lograrlos" (p. 758), apuntando, además, que la base para la precisión de esos objetivos es el análisis de las necesidades de capacitación.

- Principio del desarrollo continuo, que estipula: "Cuanto más se aplica una empresa en pos de la excelencia administrativa, más requiere que los administradores prosigan con su desarrollo personal" (p. 758).

Mace (1990), por su parte, plantea algunas ideas generales que son de valor para orientar la formación de los directivos y, por consiguiente, para la formación especializada de los directores escolares. Destaca el "aprender actuando" como "el método principal y básico para desarrollar las dotes y las capacidades de los ejecutivos" y la necesidad de "planear las fases de conocimiento laboral para los ejecutivos en potencia de nivel superior” (pp. 190-191).

Cuesta (2009), que propone "principios para la organización que aprende", plantea algunas ideas de sumo interés: a) la consideración de la formación continua como sustento esencial de la gestión de competencias, b) la concepción de la formación para más de un puesto de trabajo, para laborar en grupos o equipos y para una cultura organizacional, c) la íntima vinculación que ha de tener la formación con el proyecto de 
vida de las personas, d) la necesidad de establecer un ciclo de formación que incluye la determinación de las necesidades, la elaboración del plan de formación, su ejecución y su evaluación, y; e) la conveniencia de la definición de planes de carrera.

Asimismo, en trabajos que abordan la formación del director escolar, como objeto específico, se pueden encontrar "ideas rectoras" que resultan de gran utilidad a los fines de establecer principios de su formación especializada, en tanto ofrecen pautas teóricas y metodológicas que permiten explicar dicho proceso y pueden servir de guía para su realización práctica. Entre esas “ideas rectoras” vale la pena destacar:

- La importancia de comenzar la preparación para la dirección escolar durante la formación inicial (de pregrado) del docente, reconocida por autores como Brito y Plasencia (2011); Del Toro, Valiente y González (2014); Fernández (2002), Gómez (2010); Valiente, González y Del Toro (2013). Al respecto Gómez (2010) ha señalado:

El aprendizaje sobre las características, naturaleza de las organizaciones, el trabajo en equipo, la participación o la toma de decisiones, permitirá al profesorado incorporarse a la organización con una mayor predisposición hacia tareas colaborativas, asi como participar de manera más productiva en tareas diferentes a las docentes desde un primer momento. ( $\mathrm{p}$. 169)

- La necesidad de la integración entre una formación inicial y una formación permanente (continua), como etapas que han sido denominadas con diversos términos: a) formación inicial y permanente (Gómez, Oliva y López, 2014; Sáenz y Debón, 1998); b) formación inicial y formación continuada (Caballero, 2003); c) formación inicial, formación para la función y formación en la función directiva (Gairín, 1995); d) sucesión de liderazgo, preparación, selección, inducción y desarrollo en el servicio (Bush, 2008); y e) formación pre-servicio, inducción y formación continua (Weinstein y Hernández, 2014).

- La concepción de la formación del director como específica y diferenciada de la del docente, dadas las particularidades que distinguen las tareas que cada uno debe desempeñar, idea sostenida por numerosos autores como Caballero (2003), Gairín (1995) y Sáenz y Debón (1998). Sobre el particular Bush (2008) ha dicho:
La perspectiva tradicional en muchos países es que el director de la escuela y el personal de mayor categoría solamente necesitan estar calificados y ser maestros de experiencia. Sin embargo, ahora emerge el reconocimiento de que el liderazgo es una profesión paralela si no aparte y requiere de preparación especifica. (...) La designación de los directores de escuela sin preparación específica es un riesgo y no debemos jugar con la educación de los niños. (p. $\mathrm{xi})$

- La concepción de la formación a partir de considerar la condición de adultos de los directores como sujetos de aprendizaje, que permita aprovechar toda su educación y experiencia previa, la aplicación del conocimiento personal y la capacidad de liderar y dirigir, como ha sido planteado, entre otros autores, por Murillo, Barrio y Pérez-Albo (1999) y Valiente (2001).

- La conveniencia del diagnóstico de necesidades como condición previa a la realización del proceso de formación, criterio ampliamente reconocido por toda la comunidad de especialistas e investigadores del tema.

- La contextualización del contenido de la formación considerando, entre otros elementos, la multidisciplinariedad de su cuerpo teórico, el modelo de institución 
educativa y de dirección establecidos, la realidad del lugar de trabajo, la introducción de resultados de investigaciones que favorezcan la mejora escolar, las fuerzas externas, la dinámica y el cambio que tiene lugar en el entorno; así como el desarrollo tecnológico y las políticas que le conciernen.

- La consideración de prioridades temáticas en el contenido, relativas a la preparación para: el ejercicio de un liderazgo eficaz, la gestión de recursos humanos, el despliegue de los roles y funciones específicas que competen a la APD del director escolar, el desarrollo de habilidades y capacidades directivas genéricas, la promoción del cambio, el aprendizaje de técnicas de gestión, el desarrollo de habilidades y actitudes sociales, entre otras expuestas en una multiplicidad de trabajos.

- La necesidad de una concepción metodológica especial en el proceso pedagógico de la formación que favorezca el reforzamiento del vínculo entre los directivos en formación, el intercambio de experiencias y posibles colaboraciones posteriores. En tal sentido se reconoce la "formación centrada en el análisis de las prácticas" como un enfoque especial a considerar (López y Sánchez, 1996; Nicastro, 2014; Poggi, 2001). De igual modo, se han identificado enfoques, métodos y formas de organización especializadas como son: los estudios de casos, el aprendizaje basado en problemas, los talleres interactivos, el aprendizaje en el terreno, la consultoría, los concursos de planes de negocio y el aprendizaje en el puesto, utilizados en programas de maestría en Administración de Negocios (MBA), Administración Pública (MPA) y Administración de Negocios con énfasis en Educación (MBAEd), desarrollados por universidades de Estados Unidos, Canadá, Reino Unido, Francia, Italia, Holanda, Suiza, España, China, Hong Kong, Corea del Sur y Tailandia, reconocidas internacionalmente por la calidad de sus programas de postgrado en el área de Administración. (Hallinger y Lu, 2013)

- El empleo de la evaluación como mecanismo para valorar el desarrollo y los resultados de experiencias de formación en curso y para la valoración de las habilidades, conocimientos y capacidad de desarrollo adquiridos por los participantes, como recomiendan Gairín (1995), Poggi (2001) y Valiente (2001), entre muchos autores. En referencia a este asunto, Lumby, Walker, Bryant, Bush y Björk (2009) recomiendan: "Promover mayor sensibilidad sobre la necesidad de que los modelos de evaluación de programas de preparación sean contextual y culturalmente relevantes" (p. 188).

- La necesidad de lograr una adecuada interrelación entre teoría y práctica. Sobre esta exigencia de la formación de los directores escolares, acerca de la que existe un amplio reconocimiento. Bush y Jackson (2002) enfatizan en la necesidad de una adecuada articulación entre teoría y práctica que permita a los directores incorporar los resultados de investigaciones al momento de tomar decisiones, y saber vincular sus problemas cotidianos con lo expuesto por diferentes teorías.

- La necesidad de retener, atender y desarrollar, con intencionalidad, el talento profesional de jóvenes con posibilidades para el liderazgo en las instituciones escolares. El reconocimiento de esta necesidad va siendo creciente y se concreta en experiencias específicas en países como Cuba, por medio del movimiento de la Reserva Especial Pedagógica, e Inglaterra, donde se aplica el Programa Nacional de Planificación de la Sucesión para las Escuelas (Rhodes y Brundrett, 2014). En 
el caso de Cuba, el movimiento de la Reserva Especial Pedagógica, sustentado en la voluntariedad de sus integrantes, agrupa a aquellos docentes jóvenes con potencialidades para el ejercicio de cargos directivos en los distintos niveles de la estructura del sistema educativo, especialmente en los centros escolares. Sus integrantes son detectados y se incorporan al movimiento desde la formación inicial de pregrado en las carreras pedagógicas. Su razón de ser esencial es la preparación de sus miembros para la asunción de las referidas tareas directivas.

Otro antecedente importante a los fines de formular una propuesta de principios de la FEDE, se encuentra en un conjunto de trabajos de autores cubanos que han enunciado principios y otros postulados teóricos de carácter general, relativos a la formación y superación de directivos, docentes y otros grupos de recursos laborales, entre los que se incluyen algunos específicos para la formación de los directores escolares (cuadro 1).

Cuadro 1. Algunas propuestas de principios y postulados teóricos generales relativos a la formación de directivos, docentes y otros grupos de recursos laborales

PRinCiPIOS DEL CAMbio EDUCATIVO (García et al., 1996) (Válidos para los procesos de autoperfeccionamiento docente)

- Del aumento de la participación activa y

- Del desarrollo y estimulación de la creatividad

democrática

- De la motivación

- Del cambio de significado de la actividad

Principios de EdUCaCión AvanZada (Añorga, 2001)

- De la relación entre la pertinencia social, los objetivos, la motivación y la comunicación

- Del vínculo entre la racionalidad, la creatividad y la calidad del resultado

- Del carácter científico del contenido, la investigación e independencia cognoscitiva y la producción de nuevos conocimientos

- De la relación entre las formas, las tecnologías y su acreditabilidad

- Del vínculo de la teoría con la práctica en la formación de valores

- Del enfoque de sistema y sus expresiones ramales, sectoriales, territoriales y comunitarios

- De la condicionalidad entre la formación de pregrado, la básica y la especializada REGULARIDADES DE LA CONCEPCIÓN SISTÉMICA DE LA SUPERACIÓN DE LOS DIRIGENTES EDUCACIONALES (Valiente, 2001)

- Entre el diseño, la pertinencia y la efectividad del proceso de superación

- Entre la política para el diseño y ejecución de la superación y la concepción metodológica y el clima afectivo en el proceso de enseñanza - aprendizaje

- Entre el carácter, contenido y contexto multivariado de la actividad directiva y el enfoque multidimensional e interdisciplinario de los objetivos y el contenido de la superación

- Entre la modelación de la profesionalidad, la proyección individual de desarrollo del directivo, la personalización del currículo y la evaluación del desempeño

\section{PRINCIPIOS QUE RIGEN LA SUPERACIÓN DE LOS DIRECTORES DE ESCUELA (Valle, 2001)}

- De la atención diferenciada

- De la búsqueda colectiva de soluciones a problemas modelados de la práctica escolar

- Del intercambio de experiencias

- De la discusión de soluciones y la comprobación práctica de las mismas

\section{REGULARIDADES DE LA DIRECCIÓN DE LA SUPERACIÓN DE LOS DOCENTES DE LA ESCUELA} SECUNDARIA BÁSICA (Castillo, 2004)

- Relación entre interdisciplinariedad, desempeño profesional e integración

- Relación entre dirección de la superación desde la escuela, el trabajo cooperado en el grupo y la evaluación del desempeño profesional como variable de la evaluación de impacto.

PRINCIPIOS DE LA SUPERACIÓN PROFESIONAL (Ginoris et al., 2005)

- Diagnóstico continuo

- Sistematicidad

- Carácter sistémico

- Carácter integral

- Dirección social
- Diferenciación

- Trabajo individual y grupal

- Retroalimentación

- Intercambio profesional

Fuente: Elaboración propia. 
Cuadro 1. Algunas propuestas de principios y postulados teóricos generales relativos a la formación de directivos, docentes y otros grupos de recursos laborales. Continuación

PrinCIPIOS DE la PrePaRACIÓN Y SUPERACIÓN DE loS CUADROS DEL ESTADO Y DEL GOBIERNO Y SUS RESERVAS (Consejo de Estado, 2010)

- Del carácter general de la aplicación de la preparación y superación de los cuadros y sus reservas (en todos los sectores y niveles), acorde a la Política del Partido Comunista de Cuba, del Estado y del Gobierno.

- De la preparación y superación de los cuadros y sus reservas como un proceso continuo (permanente), planificado (individualmente) y gradual (escalonado) se inicia durante el ejercicio de su profesión, con la identificación de sus potencialidades y capacidades para el liderazgo.

- De la correspondencia de la preparación y superación de los cuadros y sus reservas con la planificación del país a largo plazo.

- De la preparación teórica y práctica, la articulación armónica de los componentes de su contenido y el enfoque político- ideológico de la formación.

- Del diseño de la formación en correspondencia con el perfil del cargo, el resultado de la evaluación del desempeño y la determinación de las necesidades de aprendizaje.

- De la contextualización del contenido de la preparación a las condiciones generales y particulares de cada nivel, a las transformaciones que se produzcan y a las necesidades de los cuadros.

- De la responsabilidad y el compromiso del cuadro con su superación, en especial con su autopreparación.

- De la responsabilidad máxima que compete a los jefes de cada nivel de dirección con el desarrollo de la estrategia de cuadros

- El término "cuadros" se utiliza para nombrar a aquellos “(...) trabajadores que (...) son designados o electos para ocupar cargos de dirección en la función pública, en los sistemas de las organizaciones económicas y unidades presupuestadas del Estado y del Gobierno" (Consejo de Estado, 1999, p. 1).

\section{OTRAS PROPUESTAS}

- Del vínculo entre los referentes orientadores, la progresión planeada y la evaluación de la formación previa del director de centro docente en el contexto del movimiento de la Reserva Especial Pedagógica. (González, 2009)

- Del vínculo entre la orientación referencial contextualizada, el diseño y ejecución contextualizados y la progresión cualitativa de la formación permanente de los directores escolares zonales del sector rural (Santiesteban, 2011)

- De la unidad entre la pertinencia de los referentes de orientación, la integralidad en la proyección, el enfoque pedagógico y la sistematicidad de la evaluación. (Tamayo, 2011)

Fuente: Elaboración propia.

Una mirada particular a cada propuesta permite apreciar que en varios casos estas ponen el énfasis en la orientación del proceso pedagógico que concierne a la formación (Castillo, 2004; García et al., 1996; Valle, 2001) y otras, la mayor parte, se enfocan más a la orientación del proceso de dirección que le atañe (Añorga, 2001; Consejo de Estado, 2010; Ginoris et al., 2005; González, 2009; Santiesteban, 2011; Tamayo, 2011; Valiente, 2001). Lo anterior tiene que ver con el propósito que animó el planteamiento de cada conjunto de principios o regularidades, que forman parte de propuestas más generales, en la que la intencionalidad pudo estar centrada, de manera particular, en uno u otro de los procesos (pedagógico y directivo) inherentes a la formación de directivos educacionales, docentes u otros segmentos de recursos humanos.

El análisis permitió precisar, asimismo, que los elementos más recurrentes a que aluden los principios y regularidades contenidos en las propuestas estudiadas están asociados a:

- La necesidad de contar con referentes de orientación para el diseño y ejecución de la formación que aseguren su pertinencia, como pueden ser los resultados 
del diagnóstico de necesidades, la modelación de cualidades ideales y el contenido de la APD, fundamentalmente.

- La atención diferenciada a los participantes en la formación.

- La contextualización del contenido de la formación y componentes que debieran ser considerados en el mismo.

- La sistematicidad de la evaluación en el proceso formativo.

- El enfoque sistémico, carácter progresivo e integralidad de la proyección y ejecución de la formación.

- El enfoque metodológico del proceso de formación.

- La vinculación entre teoría y práctica en la concepción de la formación.

La identificación de estos elementos de mayor recurrencia en el conjunto de propuestas estudiado, ofrece un marco teórico general de gran valor para la orientación (teórica y metodológica) de la FEDE, por lo que se constituyen en un referente principal para la propuesta de principios.

De igual forma, debe destacarse el valor de ciertos principios que, aunque no tienen una presencia recurrente, resultan referentes específicos de gran utilidad. Tal es el caso del "principio de la condicionalidad entre la formación de pregrado, la básica y la especializada” planteado por Añorga (2001), que constituye el precedente principal para el planteamiento de un principio que oriente la relación que debe darse entre la preparación para la dirección escolar que recibe el educador en su formación inicial de pregrado y la concepción y el contenido formativo de las etapas que incluye la FEDE (inicial y permanente).

\section{Metodología}

La formulación de un sistema de principios de la FEDE, para explicar teóricamente dicho proceso y ofrecer la orientación metodológica que viabilice su realización en la práctica, constituyó el objetivo de una tarea de investigación, desarrollada como parte del Proyecto de Formación del Director Escolar que encaminan los autores, cuya fundamentación teórica, concepción metodológica y resultados se exponen en este artículo.

La enunciación de nuevos principios en el sistema teórico de cualquier ciencia resulta siempre un asunto muy debatido, que exige el cumplimiento de una estrategia metodológica que asegure el máximo rigor científico y evite caer en una posición especulativa, carente de fundamentos convincentes.

Al respecto, Engels (como se citó en Valcárcel, 1998) planteó que de los principios no se parte “...sino que a los principios se llega, y una vez que la experiencia científica acumulada es lo suficientemente válida como para la formulación de un principio, este se convierte en premisa indispensable para el futuro desarrollo de cualquier proceso" (p. 111).

En consonancia con lo antes planteado, la estrategia metodológica seguida para alcanzar el objetivo propuesto en la investigación fue la de sistematización de la experiencia teórica precedente relativa a la formación de los directores escolares, otros profesionales de la educación, directivos empresariales y otros grupos de recursos laborales, mediante 
el examen exhaustivo de la literatura disponible, para determinar en qué medida las propuestas existentes satisfacían las expectativas de la investigación; así como precisar ideas rectoras y pautas de carácter general que sirvieran de soporte al sistema de principios que debía formularse.

Para viabilizar la estrategia asumida se siguieron los siguientes pasos metodológicos sugeridos por Castañeda (2007): a) Análisis del grado de satisfacción de los sistemas de principios y otros postulados teóricos generales existentes, a la solución del problema investigado; b) Valoración de la consideración de los sistemas de principios existentes en las diferentes propuestas; c) Adopción de los principios que se consideran pertinentes para orientar teórica y metodológicamente la FEDE; d) Planteamiento de nuevos principios que permiten explicar nuevas relaciones y cualidades esenciales de la citada formación ; y e) Formulación de los principios que conforman la propuesta, considerando la definición de sus postulados esenciales, que expresan el modo en que explican la esencia del proceso de formación especializada, y el planteamiento de los requerimientos (implicaciones) que exige su utilización práctica y coadyuvan al cumplimiento de su función metodológica.

El proceso de sistematización y formulación del sistema de principios se desarrolló, básicamente, a partir de la utilización de métodos teóricos propios de la investigación educacional como el análisis y la síntesis, la inducción y la deducción, la modelación y el enfoque de sistema, empleados en estrecha unidad y correlación.

\section{Resultados}

A partir de la revisión analítica de las obras consultadas, se sintetizó el conjunto de ideas rectoras y elementos recurrentes resumidos en el apartado correspondiente a la fundamentación teórica. Se concluyó que estos, como totalidad, ofrecen un marco teórico general que se constituye en el referente principal para la propuesta de principios que se expone como resultado de la tarea de investigación.

Asimismo, se consideró que las propuestas más cercanas al objeto de estudio no satisfacían plenamente las expectativas de la investigación. No obstante, se asumió el valor de ciertos principios o postulados generales que, aunque no tienen una presencia recurrente, resultan referentes específicos de gran utilidad para la definición de principios particulares de la propuesta a modelar.

En consecuencia con lo anteriormente analizado, se proponen cinco principios de la FEDE a los que corresponde la función de orientar teórica y metodológicamente su diseño y ejecución, considerando su naturaleza pedagógica y directiva.

\subsection{Principio del vínculo entre la formación inicial del educador y la formación especializada (inicial y permanente) para la dirección escolar}

El proceso de formación para la dirección escolar se ha de iniciar durante la formación inicial (de pregrado) del profesional de la educación, en la que, junto a la preparación que recibe para la dirección del proceso educativo de los alumnos, debe adquirir conocimientos, desarrollar habilidades y formarse en valores que le posibiliten la dirección de otros procesos que tienen lugar en la institución escolar, cuyo contenido rebasa los marcos de su accionar en el grupo (o grupos) en que se desempeñará como docente. 
Aun cuando la actividad pedagógica profesional del docente tiene una naturaleza directiva, no basta con que el futuro educador sea preparado para el desempeño de las funciones y roles que conciernen específicamente a dicha actividad. Se requiere que, de manera intencionada, el modelo curricular de la formación incluya contenidos que lo preparen para desplegar funciones y roles que corresponden al contenido de la actividad profesional de otros cargos que forman parte de la estructura organizativa de la escuela (subdirectores, jefes de ciclos, jefes de grados, jefes de departamentos, profesores principales, etc.).

De este modo, la formación de pregrado del profesional de la educación se erige en el basamento necesario para la formación especializada (inicial y permanente) del director escolar, en la que el énfasis de la formación se enfoca hacia la preparación para el desempeño de las funciones y roles que corresponden al contenido de su actividad profesional de dirección. La calidad de la formación lograda en el pregrado constituye, por tanto, una variable esencial para una mayor eficacia en la formación especializada.

El cumplimiento de este principio tiene, entre otras implicaciones:

- La consideración de la preparación para la dirección escolar como una estrategia curricular (eje transversal) de la formación inicial del profesional de la educación, que sirva de marco a la inclusión y tratamiento de contenidos seleccionados y el desarrollo de actividades específicas, dirigidos a ese propósito, de un modo gradual, en los diferentes años de la carrera.

- La utilización del contenido que incumbe a la APD de los diferentes cargos y responsabilidades presentes en la estructura de dirección de la institución educativa, como un referente importante para determinar el contenido de la preparación para la dirección escolar, durante la formación de pregrado.

- La retroalimentación sistemática, por medio de acciones de evaluación, del contenido formativo dirigido a la preparación para la dirección escolar en el diseño curricular de la formación inicial del educador.

- El diagnóstico preciso de la preparación para el ejercicio de las funciones directivas escolares que recibió el futuro directivo escolar durante su formación de pregrado, como un referente de orientación fundamental en la definición del contenido de la formación especializada, particularmente en su etapa inicial.

\subsection{Principio de la condicionalidad entre los referentes orientadores y la} pertinencia social de la formación

La pertinencia social de la formación especializada de los directores escolares se puede definir como la capacidad de respuesta de las acciones (programas, sistemas, estrategias, etc.) de formación (inicial o permanente), que se diseñan y desarrollan, a las necesidades sociales relevantes presentes y perspectivas y a las exigencias que de ellas se derivan para la política educacional, el funcionamiento del sistema educativo, las instituciones escolares, y el proceso de dirección de estas últimas.

Dicha pertinencia sólo puede alcanzarse si la concepción del proceso de formación toma en cuenta, y aprovecha al máximo, la información que sobre dichas necesidades proveen los referentes orientadores de la formación. La utilización eficaz de esta información asegura el carácter intencionado y consciente del proceso formativo. 
Los referentes orientadores de la formación son las fuentes proveedoras de información que se utiliza como insumo para su diseño. La información obtenida desde dichas fuentes está relacionada, entre otros elementos, con: a) las directrices (políticas) que orientan la formación; b) lo que debe hacer el director como parte de su labor directiva: su actividad profesional de dirección; c) las cualidades "ideales" que debería reunir el director para desarrollar con efectividad el contenido de su APD y; d) el estado real de su preparación y desempeño profesional. La pertinencia de la concepción del proceso de formación de los directores escolares está condicionada por la suficiencia y oportunidad de la información que se tenga sobre los elementos antes mencionados y de su empleo efectivo en el diseño y ejecución de dicho proceso.

El cumplimiento de este principio atañe tanto al proceso pedagógico de la FEDE, para cuya dirección sus conductores deben considerarlos en la concepción de las acciones que tienen a su cargo desarrollar; como al proceso de dirección que es inherente a dicha formación, en el que los gestores deben tomarlos en cuenta para la concepción de la formación del grupo de sujetos a quienes va dirigido un programa específico de formación.

Son implicaciones importantes del cumplimiento de este principio:

- La obtención de información desde todos los referentes, dada la especificidad de la que cada uno de ellos aporta: exigencias sociales al proceso directivo y al desempeño de sus conductores, el contenido de la actividad directiva escolar, las cualidades que deben poseer quienes la llevan a cabo y, el estado de la preparación y el desempeño de quienes la ejercen o ejercerán.

- La triangulación de la información obtenida desde todos los referentes orientadores, como condición previa necesaria para la definición de los objetivos y el contenido de la formación especializada.

- La modelación de procedimientos o metodologías que viabilicen la obtención de la información desde los referentes orientadores.

\subsection{Principio del enfoque sistémico, progresivo y planeado de la formación}

Este principio, que compete particularmente al proceso de dirección de la formación de los directores escolares, parte de reconocer que la formación de los directivos escolares tiene un carácter específico y diferente de la formación de los docentes, que es un proceso complejo, a largo plazo, que requiere de una variedad de recursos e involucra una diversidad de actividades.

El principio postula que la FEDE ha de concebirse desde el enfoque de sistema, como un proceso continuo, de desarrollo progresivo (escalonado, gradual, por etapas) y compromiso personal, que exige ser planeado para asegurar el éxito de su realización.

Su materialización en el diseño y ejecución de la formación especializada de los directores escolares tiene, entre otras implicaciones:

- La articulación adecuada entre el contenido de la preparación para la ejecución de tareas directivas en la institución escolar que tiene lugar durante la formación del educador, en el pregrado, y la formación especializada para la dirección escolar. 
- El logro de una relación sistémica entre la formación inicial y permanente, como etapas de la formación especializada para la dirección escolar.

- La planeación en sistema de las actividades (colectivas e individuales) que conforman el diseño de las acciones de formación (estrategias, programas o sistemas de formación).

- El establecimiento de niveles en las etapas de la formación especializada (por los que han de transitar los sujetos participantes), en los que se prevén objetivos y contenidos cuya complejidad aumenta de manera creciente.

- La planeación individual de la formación como expresión de la conjunción entre las exigencias sociales al desempeño del director escolar y las necesidades individuales de cada sujeto participante, sin dejar de considerar su proyecto personal de vida.

- La planeación colectiva de la formación como respuesta a las exigencias sociales y a las necesidades grupales de los sujetos participantes en las acciones de formación.

\subsection{Principio de la relación entre el contenido contextualizado de la formación, el trabajo cooperado en grupo y la atención diferenciada en el proceso formativo}

El contenido de este principio está más vinculado al contenido pedagógico del proceso de FEDE. Sus implicaciones están más relacionadas, por tanto, con la concepción metodológica de las actividades formativas y conciernen, básicamente, a la selección del contenido a abordar en las mismas, a los métodos y formas organizativas para su tratamiento en el contexto grupal y a la necesidad de ofrecer un espacio adecuado para la satisfacción de las necesidades educativas individuales de los participantes y el despliegue de sus potencialidades.

La selección del contenido en el proceso de formación del director escolar debe asegurar su contextualización. Ello significa que ha de satisfacer tanto las exigencias sociales a la dirección de las instituciones escolares como las necesidades grupales e individuales de los participantes. Debe propiciar, además, la búsqueda de respuestas a los problemas más apremiantes que están presentes en el entorno laboral de los sujetos en formación.

Una selección pertinente del contenido de la formación presume, por consiguiente, una utilización oportuna y suficiente de la información que proveen los referentes orientadores de la formación y el conocimiento profundo de la problemática del contexto laboral de los participantes en las acciones de formación.

La contextualización del contenido de la formación no podrá ser, por tanto, un proceso estático, sino flexible, en el que las prioridades temáticas consideradas en los programas de formación sean sometidas a una actualización sistemática.

La dinámica del proceso pedagógico durante las acciones formativas debe contribuir a elevar la preparación individual de los participantes, en los objetivos previstos, a partir del aprovechamiento de las posibilidades que ofrece el trabajo cooperado grupal para la solución de problemas y el planteamiento de tareas que resulten significativas para todos. 
El trabajo cooperado en grupo, para el tratamiento del contenido contextualizado de la formación y la atención diferenciada en el proceso formativo, exige entre otros requerimientos:

- La concepción de la formación a partir de considerar la condición de adultos de los sujetos de aprendizaje (con todas las implicaciones que ello supone) que permita aprovechar su preparación y experiencia previa.

- La consideración de la formación como un proceso de cambio, lo que implica la utilización de los principios que promueven su desarrollo eficaz.

- La concepción de la formación como un proceso de autoperfeccionamiento docente, entendido "como una actividad autotransformadora que presupone el cambio del docente centrado en el dominio y comprensión profunda de los fines y naturaleza de su actuación profesional, incluyendo los mecanismos que facilitan su cambio sistemático y su autoanálisis" (García et al., 1996, p. 19).

- La creación del compromiso y el sentido de pertenencia individual con el grupo y con la necesidad de buscar soluciones en el marco del proceso de formación, mediante el trabajo cooperado, a las problemáticas que limitan el desempeño individual y grupal para la labor de dirección escolar y pueden afectar (o afectan) sus resultados.

- La creación de la capacidad de trabajo colaborativo en el grupo, lo que implica el establecimiento y consolidación de relaciones grupales estrechas y eficaces, que abran el camino a la colaboración.

- La concertación colectiva de las actividades a desarrollar en el proceso de formación, lo que incluye la definición de los objetivos a alcanzar y la selección del contenido a abordar en dichas actividades.

- La combinación de formas organizativas que aseguren una adecuada interrelación entre teoría y práctica.

- La utilización de métodos y procedimientos de enseñanza-aprendizaje especiales que posibiliten el intercambio de las experiencias propias, el conocimiento personal de los miembros del grupo y la "confrontación con el conocimiento intelectual” (Castillo, 2004), durante el tratamiento del contenido de las actividades de formación.

\subsection{Principio de la relación entre la sistematicidad de la evaluación y el mejoramiento cualitativo del proceso y los resultados de la formación}

La evaluación se constituye en un medio esencial para la gestión de la calidad del proceso de formación del director escolar. La sistematicidad de su aplicación supone considerarla, desde su propia gestación, en todos los momentos del proceso formativo, en cada uno de los cuales cumplirá funciones específicas.

La evaluación tendrá la misión de producir información caracterizadora y valorativa sobre el diseño de la formación (evaluación preactiva), el proceso de su ejecución (evaluación interactiva) y sus resultados (evaluación postactiva). Entre estos últimos tienen un lugar relevante los que competen a las transformaciones (en el corto, mediano y largo plazo) que se producen en la preparación y el desempeño individual de los sujetos 
participantes, como consecuencia del efecto de las acciones formativas, y aquellos que están relacionados con el impacto de la formación en el desarrollo y desempeño organizacional.

La aplicación de este principio ha de estar también presente en el proceso pedagógico de la FEDE, del cual la evaluación es un componente reconocido que tiene entre sus funciones aportar información sobre la eficacia (cumplimiento de los objetivos) de las acciones de formación, que debe ser utilizada por gestores (directivos superiores) y conductores (facilitadores) para actuar en su mejoramiento.

El cumplimiento de este principio presume:

- El diseño del proceso de evaluación en cada uno de los momentos en que esta debe efectuarse (antes, durante y después del desarrollo de las acciones formativas), lo que supone proyectar sus objetivos, definir su contenido, determinar los métodos, instrumentos, fuentes y mecanismos para la recogida de datos y la obtención de información, entre otros requerimientos.

- La utilización de la información producida por la evaluación en la adecuación y el rediseño de los programas, sistemas y acciones de formación previstas; así como de los planes individuales de formación y desarrollo de los directores implicados.

\section{Discusión y Conclusiones}

La expansión de experiencias formalizadas y otras acciones prácticas dirigidas a la formación de los directores escolares y el crecimiento significativo de los estudios relacionados con este objeto, que se ha producido en paralelo, han conllevado a una notable acumulación de conocimientos que requieren ser sistematizados.

La sistematización de este amplio arsenal de saberes teóricos y prácticos, desde la perspectiva de la ciencia, supone el planteamiento y argumentación de relaciones esenciales que son inmanentes al proceso de formación del director escolar como objeto de estudio.

La propuesta de principios antes presentada, sustentada en la sistematización de la experiencia (práctica e investigativa) precedente, representa un intento que puede ser valioso para el mejor conocimiento del proceso estudiado a partir de la construcción de un sistema teórico ordenado, aun no existente. Hay que considerar que una gran parte de los conocimientos atesorados proviene de estudios en que la estrategia de investigación ha estado basada en el estudio de casos, y por tanto, no se conciben como conocimientos generalizables.

Los principios expuestos constituyen un componente fundamental de la concepción teórico-metodológica de la FEDE que se modela desde el Proyecto de Investigación que desarrollan los autores. Coadyuvan a ofrecer un marco teórico y metodológico que permite la realización pertinente de los procesos (pedagógico y directivo) en que se concreta dicha formación. Poseen, por consiguiente, una función transformadora por cuanto su aplicación consecuente contribuye al mejoramiento cualitativo de los procesos que son inherentes a este tipo particular de formación. 
En tanto son generalizaciones teóricas derivadas de la sistematización de la "experiencia científica acumulada”, cumplen, a la vez, una función gnoseológica y una función metodológica. Develan relaciones esenciales que son explicativas del contenido de dicho proceso formativo y constituyen, por consiguiente, uno de los cimientos principales de su basamento teórico. De otro lado, al constituir "premisa indispensable para el futuro desarrollo de cualquier proceso", ofrecen orientaciones indicativas de las implicaciones que tiene la realización de la FEDE como proceso práctico.

A diferencia de otras propuestas precedentes que privilegian la atención de alguno de los procesos o elementos específicos que conciernen a la formación del director escolar, el sistema de principios formulado aborda en su integralidad tanto al proceso pedagógico como al proceso directivo que atañen a dicha formación.

Los principios propuestos tienen también un carácter socio-histórico concreto y son, por tanto, susceptibles de reconceptualización y adecuación en las condiciones de cada contexto, en consonancia con las demandas que cada momento histórico le plantea a la formación de los directores escolares, devenidas de las crecientes exigencias sociales a los sistemas educativos y las instituciones que los conforman.

\section{Referencias}

Añorga, J. (2001). La Educación Avanzada. La Habana: Academia.

Brito J. y Plasencia, A. (2011). Realidad y perspectiva de las competencias para el ejercicio directivo en Cuba. En J. Gairín y D. Castro (Eds.), Competencias para el ejercicio de la dirección de instituciones educativas. reflexiones y experiencias en Iberoamérica (pp. 67-78). Santiago: Red de Apoyo a la Gestión Educativa.

Bush, T. (2008). Leadership and management development in education. Londres: Sage.

Bush, T. y Jackson, D. (2002). A preparation for school leadership. International perspectives. Educational Management Administration E' Leadership, 30(04), 417-429.

Caballero, J. (2003). La necesidad de formación de los directores escolares en la Comunidad Autónoma de Andalucía. Revista Electrónica Interuniversitaria de Formación del Profesorado, 6(1), 1-12.

Castañeda, A. (2007). Modelación de la formación de habilidades manuales para la tornería en los estudiantes de las especialidades de la familia mecánica de la Educación técnica y profesional. Tesis doctoral. Instituto Superior Pedagógico José de La Luz y Caballero. Holguín (Cuba).

Castillo, T. (2004). Un modelo para la dirección de la superación de los docentes desde la escuela secundaria básica. Tesis doctoral. Instituto Central de Ciencias Pedagógicas. La Habana (Cuba).

Consejo de Estado de la República de Cuba (1999). Decreto ley 196. Sistema de trabajo con los cuadros del estado y del gobierno. La Habana: Autor.

Consejo de Estado de la República de Cuba (2010). Perfeccionamiento del sistema de trabajo con los cuadros y sus reservas. Anexo 6. La Habana: Autor.

Cuesta, A. (2009). Tecnología de gestión de recursos humanos. La Habana: Félix Varela.

Del Toro, J., Valiente, P. y González, J. (2014). La preparación para la dirección escolar en la formación inicial del docente: resultados de un estudio diagnóstico en una universidad pedagógica cubana. Holguín: Universidad de Ciencias Pedagógicas José de la Luz y Caballero. 
Fernández, M. (2002). Formar para la dirección escolar: por qué, cuándo, cómo... Revista de Currículum y Formación del profesorado, 6(1-2), 1-12.

Gairín, J. (1995). Estudio de las necesidades de formación de los equipos directivos de los centros educativos. Madrid: CIDE.

García, L., Valle, A. y Ferrer, M. (1996). Autoperfeccionamiento docente y creatividad. La Habana: Pueblo y Educación.

Ginoris, O. (enero, 2005). Diseño estratégico de la superación profesional docente y técnicodocente de la educación general, politécnica y laboral en la provincia de Matanzas. Ponencia presentada en el Congreso Internacional Pedagogía 2005. La Habana, Cuba.

Gómez, A. (2010). La formación inicial para la dirección escolar como impulso para conseguir una dirección competente en Andalucía. Tesis doctoral. Universidad de Huelva. Huelva (España).

Gómez, A., Oliva, N. y López, J. (2014). La indefinición del modelo español de formación de líderes escolares. REICE. Revista Iberoamericana sobre Calidad, Eficacia y Cambio en Educación, 12(5), 135-150.

González, J. (2009). Modelo para la dirección de la formación previa del director de centro docente desde el movimiento de la reserva especial pedagógica. Tesis doctoral.Universidad de Ciencias Pedagógicas José de la Luz y Caballero. Holguín (Cuba).

Hallinger, P. y Lu, J. (2013). Preparing principals: what can we learn from MBA and MPA programmes? Educational Management Administration \& Leadership, 41(4), 435-452.

Koontz, H. y Weihrich, H. (2008). Administración. Una perspectiva global. La Habana: Félix Varela.

Kopnin, P. (1983). Lógica dialéctica. La Habana: Pueblo y Educación.

López, J. y Sánchez, M. (1996). Formación de directores escolares basada en el análisis de su práctica. En AA.VV, Dirección participativa y evaluación de centros. Bilbao: Ediciones Mensajero.

Lumby, J., Walker, A., Bryant, M., Bush, T. y Björk, L. (2009). Research on leadership preparation in a global context. En M. Young, G. Crow, J. Murphy y R. Ogawa (Eds.), Handbook of research on the education of school leaders (pp.157-189). Nueva York: Routledge.

Mace, L. (1990). Promoción y formación de ejecutivos. La Habana: Ciencias Sociales.

Murillo, F., Barrio, R. y Pérez-Albo, M. (1999). La dirección escolar. Análisis e investigación. Madrid: CIDE.

Nicastro, S. (2014). La formación de directores. Un modelo centrado en el análisis de las prácticas. REICE. Revista Iberoamericana sobre Calidad, Eficacia y Cambio en Educación, 12(5), 119-133

Poggi, M. (2001). La formación de directivos de instituciones educativas. Algunos aportes para el diseño de estrategias. Buenos Aires: IIPE/UNESCO.

Rhodes, C. y Brundrett, M. (2014). Reteniendo el talento de liderazgo en las escuelas. REICE. Revista Iberoamericana sobre Calidad, Eficacia y Cambio en Educación, 12(4e), 191-207.

Sáenz, O. y Debón, S. (1998). La formación del director escolar en España. Revista Electrónica Interuniversitaria de Formación del Profesorado, 1(1), 1-22.

Santiesteban, R. (2011). La dirección de la formación permanente de los directores escolares zonales del sector rural. Tesis doctoral. Universidad de Ciencias Pedagógicas José de la Luz y Caballero. Holguín (Cuba). 
Tamayo, A. (2011). La dirección de la supervisión educativa a los institutos preuniversitarios por la estructura municipal de educación. Tesis doctoral. Universidad de Ciencias Pedagógicas José de la Luz y Caballero. Holguín (Cuba).

Valcárcel, N. (1998). Estrategia interdisciplinaria de superación para profesores de ciencias de la enseñanza media. Tesis doctoral. Instituto Superior Pedagógico Enrique José Varona. La Habana (Cuba).

Valiente, P. (2001). Concepción sistémica de la superación de los directores de secundaria básica. Tesis doctoral. Instituto Superior Pedagógico José de la Luz y Caballero. Holguín (Cuba).

Valiente, P., González, J. y Del Toro, J. (2013). Fundamentos para la elaboración de una concepción teórico-metodológica de la formación especializada del director escolar. Holguín: Universidad de Ciencias Pedagógicas José de la Luz y Caballero.

Weinstein, J. y Hernández, M. (2014). Políticas hacia el liderazgo directivo escolar en Chile: una mirada comparada con otros sistemas escolares de América Latina. Psicoperspectivas, 13(3), 52-68. 